\title{
Spatially coherent diffusion of human RNA Pol II depends on transcriptional state rather than chromatin motion
}

\author{
Roman Barth ${ }^{1}$ and Haitham A. Shaban ${ }^{2,3, *}$
}

1: Department of Bionanoscience, Delft University of Technology, 2628 CJ Delft, The Netherlands.

2: Spectroscopy Department, Physics Division, National Research Centre, Dokki, 12622 Cairo, Egypt.

3: Institute of Bioengineering, Ecole Polytechnique Fédérale de Lausanne (EPFL), 1025 Lausanne, Switzerland

*: To whom correspondence should be addressed: Haitham A. Shaban. Email: haitham.shaban@epfl.ch

\begin{abstract}
Gene transcription by RNA polymerase II (RNAP II) is a tightly regulated process in the genomic, temporal, and spatial context. Transcriptionally active genes often spatially cluster at RNA Pol II foci, called transcription factories, causing long-range interactions between distal sites of the genome. Recently, we have shown that chromatin exhibits spatially long-range coherently moving regions over the entire nucleus and transcription enhances this long-range correlated DNA movement. Yet, it remains unclear how the mobility of RNA Pol II molecules are affected by transcription regulation and whether this response depends on the coordinated chromatin movement. We applied our Dense Flow reConstruction and Correlation method to analyze nucleus-wide coherent movements of RNA Pol II in living human cancer cells. We quantify the spatial correlation length of RNA Pol II in the context of DNA motion. Like DNA, we observe a spatially coherent movement of RNA Pol II molecules. However, the correlation extends over $\sim 1 \mu \mathrm{m}$, considerably less than for DNA, suggesting that spatially coherent RNA Pol II motion does not solely result from the underlying DNA motion. In contrast to DNA, inducing transcription in quiescent cells decreased the coherent motion of RNA Pol II, while the inhibition of transcription elongation by using DRB slightly increased coherent RNA Pol II motion. The spatially coherent movement of RNA Pol II domains is affected by transcriptional state and largely independent of the underlying chromatin domains. Our results suggest that RNA Pol II domains can cause the emergence of spatially correlated chromatin motion spanning several micrometers.
\end{abstract}


bioRxiv preprint doi: https://doi.org/10.1101/2022.01.19.476954; this version posted January 21, 2022. The copyright holder for this preprint (which was not certified by peer review) is the author/funder, who has granted bioRxiv a license to display the preprint in perpetuity. It is made available under aCC-BY-NC-ND 4.0 International license.

\section{Introduction}

Genome structure, dynamics, and transcription are highly coordinated to ensure proteins find the proper places on the genome in a timely manner for a correct gene expression (1). This interplay between dynamics of genome organization and transcription alters and supports the activity of the other (2). Transcription by RNA Polymerase II (Pol II) takes place for all protein-coding genes in eukaryotic genomes and is vital for many physiological processes (3). Transcription often takes place in so-called transcription factories, domains of clustered transcription factors, whose formation has been explained by liquid-liquid phase separation (4-6). These transcription factories are highly dynamic macromolecular that permit transcription initiation and elongation $(7,8)$. Transcription factories have been proposed to strongly bind DNA, likely regulatory elements, thereby constraining chromatin diffusion nuclear-wide $(9,10)$.

Advances in live cell imaging and genetic modification tools have revealed the dynamic properties of both RNA Pol II and chromatin, and their importance for transcriptional regulation. Live-cell imaging of endogenous RNA Pol II using fluorescence recovery after photobleaching uncovered several dynamic states of RNA Pol II (11). Single molecule tracking technologies have been applied to image and quantify single RNA Pol II molecules as they bind at non-specific sites throughout the genome (1214), and at a single gene (15-17). Genome-wide analysis of RNA Pol II in single living cells has been also analyzed and mapped at high resolution, using a new approach called Hi-D (18). Recently, superresolution imaging studies showed the physical relationship between RNA Pol II and chromatin clutches, but the fixation prevented the authors to deduce dynamic information of RNA Pol II $(19,20)$. In conclusion, it remains elusive if and how the diffusion of RNA Pol II is coordinated within the context of its surrounding chromatin in order to form such condensates.

Recently, we developed Dense Flow reConstruction and Correlation (DFCC), a method to study spatial and temporal long-range correlations of abundant nuclear macromolecules over entire single nuclei (21). DFCC combines light microscopy and computer vision (Optical Flow) technology to reconstruct the dynamics of bulk chromatin in diffraction-limited optical microscopy images at nanoscale resolution throughout the entire nucleus simultaneously. DFCC does not rely on the identification and tracking of single molecules, as compared to single particle tracking methods and can thus be applied to abundant nuclear proteins, allowing the estimation of their dynamics in living cells $(22,23)$. By applying DFCC to genome dynamics during transcription, we detected the formation of long-range correlated chromatin domains, extending up to several micrometres across the nucleus. These large coherent domains were reduced by the inhibition of transcription elongation (21).

Here, we apply DFCC to RNA Pol II in non-transcribing and actively transcribing cells to study the nucleus-wide coherent movements of RNA Pol II in living human cells. We find that RNA Pol II 
exhibits spatially coherent movement, which is markedly reduced upon transcription activation but only partially affected by inhibition of transcription elongation. We then calculate the spatial correlation length of RNA Pol II in the context of DNA motion. In contrast to DNA, inducing transcription in quiescent cells decreased the coherent motion of RNA Pol II. We thus conclude that the spatially coherent movement of RNA Pol II domains is largely independent of the underlying chromatin domains.

\section{Results}

To study nucleus-wide coherent movements of abundant macromolecules such as DNA, histones or RNA Pol II, we recently introduced the Dense Flow reConstruction and Correlation method (DFCC; Figure 1a) (21). DFCC applies Optical Flow on time-resolved fluorescence image series to estimate the flow field of fluorescently labeled macromolecules between successive images (Figure 1b). The displacement magnitude and direction is obtained for every pixel across the entire nucleus, which allows to compute the spatial and temporal correlation function of both flow magnitude and direction (Figure 1c). A quantitative description of these correlation functions is obtained by regression to the Matern covariance function (Figure 1c inset; Methods). To characterize flow fields of RNAP II, the correlation length $\rho_{\mathrm{c}}$ is obtained from the regression, describing how quickly correlations decay over distance (24).

We applied DFCC to investigate whether the mobility of RNA Pol II molecules exhibits coherent movement within the nucleus. Time-resolved movies of RNA Pol II (RPB1-Dendra2, subunit of RNA Pol II) in U-2 OS human osterosarcoma cell line were recorded of 150 frames with an acquisition time of $0.2 \mathrm{~s}$. Cells were grown in a medium in the absence of serum for $24 \mathrm{~h}$ in order to obtain a reference state of inactive transcription $(9,18,25,26)$. In the absence of transcription, correlated motion of RNA Pol II molecules was detected in both flow direction and magnitude, while the directional correlation length slightly increased with increasing time lag (Figure 2).

To study the correlated motions of RNA Pol II and its changes at different transcriptional stages, we imaged RNA Pol II in two different conditions, serum starved (inactively transcribing cells) and serum stimulated cells (actively transcribing). For serum starved cells, DFCC analysis showed that the correlation length for RNA pol II is time dependent with a directional correlation length increasing from $\approx 1 \mu \mathrm{m}$ to $\approx 2 \mu \mathrm{m}$ within a lag time of 10 seconds. In contrast, the directional correlation length is time independent $\left(\rho_{\mathrm{c}} \approx 1 \mu \mathrm{m}\right)$ for cells grown in normal condition (Figure $3 \mathrm{a}$ ). The difference of the directional correlation length between coherent domains could be explained by the dynamic clustering of RNA Pol II molecules at transcribed sites due to transcription activation $(1,26)$. The time-dependence of the magnitudinal correlation length may reflect the dynamic movement of RNA Pol II molecules in forming its coherent domains at their target sites. The correlation of displacement magnitudes in 
actively transcribing cells was also slightly lower compared to the transcriptionally inactive case for most time lags, yet qualitatively following a similar trend (Figure 3d). The correlation length decrease is consistent with the formation of RNA Pol II clusters at the $\beta$-actin gene in mouse embryonic fibroblast cells $(26,27)$. The non-vanishing spatial correlation of RNA Pol II molecules within the nucleus might hint at clustering of RNA Pol II during the formation of transcription factories (28-30).

Similarly, we tested how the spatial correlation of RNA Pol II responds to the inhibition of transcription elongation by using 5,6-Dichloro-1- $\beta$-D-ribofuranosylbenzimidazole (DRB). DRB pauses transcription elongation by interrupting cyclin-dependent kinase 9 (CDK9) phosphorylation $(31,32$ ). Several imaging studies of RNA Pol II proposed that transcription inhibition using DRB drug prevents the dissociation of the formed RNA Pol II clusters from the promoter-proximal paused state $(13,26,27$, 33). DRB was added to cells grown in the normal condition (serum-supplied). In comparison to serum stimulated cells, DRB treated cells showed a slight increase in directional correlation length and no change in the magnitude of correlation (Figure 3c, d). Our results suggest that the spatially coherent movement of RNA Pol II domains is affected by transcription initiation and to a smaller extent by elongation.

We then asked whether the coordinated RNA Pol II motion depends on the spatially coherent motion of chromatin (21). For this aim, the correlation length of RNA Pol II dynamics was compared to the one of chromatin (DNA labeled SiR-Hoechst) in the same cell line and conditions (Figure 4a,b). Contrary to RNA Pol II, chromatin showed an increase in correlation length $\left(\rho_{\mathrm{c}} \approx 6 \mu \mathrm{m}\right)$ in serum stimulated cells (active transcription state) and reaches a plateau after a lag time of $20 \mathrm{sec}$ (Figure 4c). Similarly, the correlation length of DNA was reduced in cells treated with DRB (plateau value $\rho_{\mathrm{c}} \approx$ $4 \mu \mathrm{m}$ and $\rho_{\mathrm{c}} \approx 0.6 \mu \mathrm{m}$ for flow direction and magnitude, respectively) (Figure $4 \mathrm{e}, \mathrm{f}$ ). Due to the fact that chromatin exhibits a considerably longer correlation length than found for RNA Pol II and contrasting responses of the correlation length upon transcriptional activation/inhibition, we conclude that the observed spatial correlation of nuclear RNA Pol II molecules cannot solely be explained by its binding to DNA. Using high-resolution imaging, recent studies also revealed the dissimilarity of RNA Pol II and DNA mobility - characterized by diffusion constant and anomalous exponent - in serum stimulated cells $(9,18)$. These studies explained the constrained motion of chromatin by the formation of transcription "hubs" or factories, which globally constrains chromatin motion. The short correlation length $\left(\rho_{c} \leq 2 \mu \mathrm{m}\right)$ of RNA Pol II likely reflects the presence of RNA Pol II clusters (transcription factories), to which freely diffusing RNA Pol II molecules are attracted. In contrast, the correlation length of chromatin in actively transcribing cells roughly 3 -fold higher, pointing to the fact that clustering of RNA Pol II modulates the spatial organization of chromatin on a scale spanning several micrometers. This finding supports the hypothesis that active alleles relocate from different positions to a shared single transcription factory to be transcribed $(34,35)$. RNA Pol II clustering, even in the 
absence of active transcription, is therefore necessary for spatial chromatin organization to reactivate transcription (36).

\section{Discussion}

In this work, we have demonstrated the capability of DFCC for detecting the spatially coordinated movement of RNA Pol II motion at nano-scale resolution. We performed a genome-wide imaging of RNA Pol II in living cells at different transcriptional states and in the context of chromatin motion. We found that RNA Pol II molecules move in a spatially correlated manner (the correlation length reaching up to $\approx 2 \mu \mathrm{m}$ ), yet its range is considerably reduced compared to the motion of chromatin. The formation of these spatially coherent RNA Pol II domains may be explained by the assembly of RNA Pol II into "transcription factories" which were previously observed in fixed cells using super-resolution techniques $(28-30)$.

We report a decrease in correlation length of RNA Pol II molecules upon transcription activation. This decrease may be due to the organization of scattered molecules into small, clustered regions such as transcription factories. Upon inhibiting transcription elongation by DRB treatment, the spatial correlation of RNA Pol II only partially recovers the non-transcribing state, hinting to a combined action of transcription initiation and elongation to the observed spatial coherence of RNA Pol II motion. A molecular picture to how RNA Pol II molecules can exhibit spatially correlated movement and how transcription initiation and elongation separately contribute to this phenomenon is currently lacking and requires further research. Nevertheless, together with the increase of coherent chromatin motion upon transcription activation, our data suggest that transcription factories persist even when transcription is inhibited, yet increase in number of participating molecules upon transcriptional activation (13) which is also concomitant with the tethering of chromatin to transcription factories $(36,37)$. 


\section{Methods}

\section{Cell culture, treatment, and imaging}

Cell culture, starvation, stimulation, treatment, and imaging are performed as described in (18). Briefly, a human U2OS osterosarcoma cell line (for DNA imaging) was maintained in Dulbecco's modified Eagle's medium including phenol red-free (Sigma-Aldrich). This cell line stably expresses RPB1 fused with Dendra2 as already described in (13). Medium was supplemented with $10 \%$ fetal bovine serum, $1 \mathrm{mM}$ sodium pyruvate (Sigma-Aldrich), Glutamax containing $50 \mu \mathrm{g} / \mathrm{ml}$ gentamicin (Sigma-Aldrich) and G418 $0.5 \mathrm{mg} / \mathrm{ml}$ (Sigma-Aldrich). Cells were cultivated at $37^{\circ} \mathrm{C}$ with $5 \% \mathrm{CO}_{2}$.

For serum starvation, cells were plated with serum-free medium and incubated for $24 \mathrm{~h}$ at $37^{\circ} \mathrm{C}$ before imaging. Before imaging, cells were mounted in L-15 medium. For stimulation, $10 \%$ fetal bovine serum was added to the medium for $15 \mathrm{~min}$.

For DRB treatment, cells were treated by adding $100 \mu \mathrm{M}$ DRB (Sigma-Aldrich) to the L-15 imaging medium that contained $10 \%$ fetal bovine serum.

For DNA staining, U2OS cells were labeled by SiR-DNA (SiR-Hoechst) at a final concentration of $2 \mu \mathrm{M}$ at $37^{\circ} \mathrm{C}$ for $30-60 \mathrm{~min}$. Before imaging, the medium was changed to L-15 medium for live imaging.

Image series of RNA Pol II was recorded as in (9). Image series of 150 frames were acquired at 5 frames per second using a Nipkow-disk confocal system. For Dendra2 excitation, a single wavelength of a $488 \mathrm{~nm}$ (Coherent) at 10\% laser power passed through a $100 \times$ oil immersion objective was applied. Images were detected on a cooled electron multiplying charge-coupled device camera (iXon Ultra 888), with sample pixel size of $88 \mathrm{~nm}$. The same imaging conditions were applied for DNA imaging, but with excitation wavelength of a $647 \mathrm{~nm}$ (Coherent) at $20 \%$ laser power.

\section{Data analysis}

Single fluorescent nuclei were manually cropped and then processed as described in (21). In brief, denoised and drift-corrected images were subject to the Optical Flow (38) and the spatial correlation of the resulting flow fields' magnitude and direction was computed for several lag times between flow fields and time-averaged. Regression of these correlation curves over distance was performed to the Whittle- Matérn covariance function (39)

$$
\mathrm{r}(\rho)=\frac{2^{1-v}}{\Gamma(v)}\left(\frac{\rho}{\rho_{\mathrm{c}}}\right)^{v} \mathrm{~K}_{v}\left(\frac{\rho}{\rho_{\mathrm{c}}}\right)
$$


where $\Gamma(\cdot)$ denotes the gamma function; $\mathrm{K}_{v}(\cdot)$ the modified Bessel function of the second type of order $v, \rho_{c}$ is the correlation length and $v$ denotes the smoothness parameter. While $\rho_{c}$ describes the long-range behavior (over which distance are two measurements correlated), the smoothness parameter $v$ describes the local, high-frequency component of the correlations (if the flow field can be described as rough or smooth, thus direction/magnitude of spatially close displacement vectors tend to (not) align on a local scale). While $v$ is also a regression parameter, we found no significant difference of $v$ across the transcriptional conditions and therefore excluded the analyses of the smoothness parameter.

Error bars of the shown correlation lengths display the standard error of the mean (SEM) of 19, 21 and 21 cells for serum-starved, serum-stimulated and DRB-treated cells, respectively.

\section{Acknowledgements}

We acknowledge support from the Pôle Scientifique de Modélisation Numérique, ENS de Lyon for providing computational resources.

Author contributions: H.A.S. designed and supervised the project. R.B. performed data analysis.

H.A.S. carried out experimental work. H.A.S. and R.B. interpreted results. H.A.S. and R.B. wrote the manuscript.

Competing interests: The authors declare that they have no competing interests.

Data and materials availability: All data needed to evaluate the conclusions in the paper are present in the paper. Additional data related to this paper may be requested from the authors. 


\section{References}

1. H. A. Shaban, R. Barth, K. Bystricky, Navigating the crowd: visualizing coordination between genome dynamics, structure, and transcription. Genome Biol. (2020), , doi:10.1186/s13059020-02185-y.

2. B. van Steensel, E. E. M. Furlong, The role of transcription in shaping the spatial organization of the genome. Nat. Rev. Mol. Cell Biol. (2019), , doi:10.1038/s41580-019-0114-6.

3. A. C. Schier, D. J. Taatjes, Structure and mechanism of the RNA polymerase II transcription machinery. Genes Dev. (2020), , doi:10.1101/gad.335679.119.

4. B. R. Sabari, A. Dall'Agnese, A. Boija, I. A. Klein, E. L. Coffey, K. Shrinivas, B. J. Abraham, N. M. Hannett, A. V. Zamudio, J. C. Manteiga, C. H. Li, Y. E. Guo, D. S. Day, J. Schuijers, E. Vasile, S. Malik, D. Hnisz, T. I. Lee, I. I. Cisse, R. G. Roeder, P. A. Sharp, A. K. Chakraborty, R. A. Young, Coactivator condensation at super-enhancers links phase separation and gene control. Science (80-. ). (2018), doi:10.1126/science.aap9195.

5. W. K. Cho, J. H. Spille, M. Hecht, C. Lee, C. Li, V. Grube, I. I. Cisse, Mediator and RNA polymerase II clusters associate in transcription-dependent condensates. Science (80-. ). 361, 412-415 (2018).

6. A. Pancholi, T. Klingberg, W. Zhang, R. Prizak, I. Mamontova, A. Noa, M. Sobucki, A. Y. Kobitski, G. U. Nienhaus, V. Zaburdaev, L. Hilbert, RNA polymerase II clusters form in line with surface condensation on regulatory chromatin. Mol. Syst. Biol. (2021), doi:10.15252/msb.202110272.

7. A. Ghamari, M. Ë. P. C. van de Corput, S. Thongjuea, W. A. Van Cappellen, W. Van Ijcken, J. Van Haren, E. Soler, D. Eick, B. Lenhard, F. G. Grosveld, In vivo live imaging of RNA polymerase II transcription factories in primary cells. Genes Dev. 27, 767-777 (2013).

8. K. Rippe, A. Papantonis, RNA polymerase II transcription compartments: from multivalent chromatin binding to liquid droplet formation? Nat. Rev. Mol. Cell Biol. (2021), , doi:10.1038/s41580-021-00401-6.

9. R. Nagashima, K. Hibino, S. S. Ashwin, M. Babokhov, S. Fujishiro, R. Imai, T. Nozaki, S. Tamura, T. Tani, H. Kimura, M. Shribak, M. T. Kanemaki, M. Sasai, K. Maeshima, Single nucleosome imaging reveals loose genome chromatin networks via active RNA polymerase II. J. Cell Biol. (2019), doi:10.1083/jcb.201811090.

10. H. A. Shaban, R. Barth, L. Recoules, K. Bystricky, Hi-D: nanoscale mapping of nuclear dynamics in single living cells. Datasets., doi:10.6084/m9.figshare.11793801.

11. B. Steurer, R. C. Janssens, B. Geverts, M. E. Geijer, F. Wienholz, A. F. Theil, J. Chang, S. Dealy, J. Pothof, W. A. van Cappellen, A. B. Houtsmuller, J. A. Marteijn, Live-cell analysis of endogenous GFP-RPB1 uncovers rapid turnover of initiating and promoter-paused RNA Polymerase II. Proc. Natl. Acad. Sci. (2018), doi:10.1073/pnas.1717920115.

12. L. Guo, L. Lei, Z.-C. Zeng, P. Zou, D. Liu, B. Zhang, L. Guo, P. Zou, D. Liu, L. Lei, B. 
Zhang, Evaluation of Spatio-Temporal Variogram Models for Mapping Xco 2 Using Satellite Observations: A Case Study in China. IEEE J. Sel. Top. Appl. EARTH Obs. Remote Sens. 8 (2015), doi:10.1109/JSTARS.2014.2363019.

13. I. I. Cisse, I. Izeddin, S. Z. Causse, L. Boudarene, A. Senecal, L. Muresan, C. Dugast-Darzacq, B. Hajj, M. Dahan, X. Darzacq, Real-time dynamics of RNA polymerase II clustering in live human cells. Science (80-. ). 341, 664-667 (2013).

14. S. Uchino, Y. Ito, Y. Sato, T. Handa, Y. Ohkawa, M. Tokunaga, H. Kimura, Live imaging of transcription sites using an elongating RNA polymerase II-specific probe. J. Cell Biol. 221, e202104134 (2021).

15. J. Li, A. Dong, K. Saydaminova, H. Chang, G. Wang, H. Ochiai, T. Yamamoto, A. Pertsinidis, Single-Molecule Nanoscopy Elucidates RNA Polymerase II Transcription at Single Genes in Live Cells. Cell (2019), doi:10.1016/j.cell.2019.05.029.

16. L. S. Forero-Quintero, W. Raymond, T. Handa, M. N. Saxton, T. Morisaki, H. Kimura, E. Bertrand, B. Munsky, T. J. Stasevich, Live-cell imaging reveals the spatiotemporal organization of endogenous RNA polymerase II phosphorylation at a single gene. Nat. Commun. (2021), doi:10.1038/s41467-021-23417-0.

17. W.-K. Cho, N. Jayanth, B. P. English, T. Inoue, J. O. Andrews, W. Conway, J. B. Grimm, J.H. Spille, L. D. Lavis, T. Lionnet, I. I. Cisse, RNA Polymerase II cluster dynamics predict mRNA output in living cells. Elife (2016), doi:10.7554/elife.13617.

18. H. A. Shaban, R. Barth, L. Recoules, K. Bystricky, Hi-D: nanoscale mapping of nuclear dynamics in single living cells. Genome Biol. 21, 95 (2020).

19. A. Castells-Garcia, I. Ed-daoui, E. González-Almela, C. Vicario, J. Ottestrom, M.

Lakadamyali, M. V. Neguembor, M. P. Cosma, Super resolution microscopy reveals how elongating RNA polymerase II and nascent RNA interact with nucleosome clutches. Nucleic Acids Res. 50, 175-190 (2022).

20. E. Miron, R. Oldenkamp, J. M. Brown, D. M. S. Pinto, C. S. Xu, A. R. Faria, H. A. Shaban, J. D. P. Rhodes, C. Innocent, S. De Ornellas, H. F. Hess, V. Buckle, L. Schermelleh, Chromatin arranges in chains of mesoscale domains with nanoscale functional topography independent of cohesin. Sci. Adv. (2020), doi:10.1126/sciadv.aba8811.

21. H. A. Shaban, R. Barth, K. Bystricky, Formation of correlated chromatin domains at nanoscale dynamic resolution during transcription. Nucleic Acids Res. 46 (2018), doi:10.1093/nar/gky269.

22. H. A. Shaban, A. Seeber, Monitoring the spatio-temporal organization and dynamics of the genome. Nucleic Acids Res. (2020), , doi:10.1093/nar/gkaa135.

23. A. A. Agbleke, A. Amitai, J. D. Buenrostro, A. Chakrabarti, L. Chu, A. S. Hansen, K. M. Koenig, A. S. Labade, S. Liu, T. Nozaki, S. Ovchinnikov, A. Seeber, H. A. Shaban, J.-H. Spille, A. D. Stephens, J.-H. Su, D. Wadduwage, Advances in Chromatin and Chromosome 
Research: Perspectives from Multiple Fields. Mol. Cell (2020), doi:10.1016/j.molcel.2020.07.003.

24. M. L. Stein, Interpolation of spatial data: some theory for kriging (Springer, 1999).

25. S. Ray, T. Panova, G. Miller, A. Volkov, A. C. G. Porter, J. Russell, K. I. Panov, J. C. B. M. Zomerdijk, Topoisomerase II $\alpha$ promotes activation of RNA polymerase I transcription by facilitating pre-initiation complex formation. Nat. Commun. 4, 1598 (2013).

26. W. K. Cho, N. Jayanth, B. P. English, T. Inoue, J. O. Andrews, W. Conway, J. B. Grimm, J. H. Spille, L. D. Lavis, T. Lionnet, I. I. Cisse, RNA Polymerase II cluster dynamics predict mRNA output in living cells. Elife. 5 (2016), doi:10.7554/eLife.13617.

27. W. K. Cho, N. Jayanth, S. Mullen, T. H. Tan, Y. J. Jung, I. I. Cissé, Super-resolution imaging of fluorescently labeled, endogenous RNA Polymerase II in living cells with CRISPR/Cas9mediated gene editing. Sci. Rep. (2016), doi:10.1038/srep35949.

28. D. R. F. Carter, C. Eskiw, P. R. Cook, Transcription factories. Biochem. Soc. Trans. (2008), , doi:10.1042/BST0360585.

29. D. Rieder, Z. Trajanoski, J. G. McNally, Transcription factories. Front. Genet. (2012), doi:10.3389/fgene.2012.00221.

30. A. Papantonis, P. R. Cook, Transcription factories: Genome organization and gene regulation. Chem. Rev. (2013), , doi:10.1021/cr300513p.

31. P. B. Sehgal, J. E. Darnell, I. Tamm, The inhibition of DRB (5,6-dichloro-1- $\beta$-dribofuranosylbenzimidazole) of hnRNA and mRNA production in HeLa cells. Cell (1976), doi:10.1016/0092-8674(76)90092-1.

32. O. Bensaude, Inhibiting eukaryotic transcription: Which compound to choose? How to evaluate its activity? Transcription (2011), doi:10.4161/trns.2.3.16172.

33. J. A. Mitchell, P. Fraser, Transcription factories are nuclear subcompartments that remain in the absence of transcription. Genes Dev. (2008), doi:10.1101/gad.454008.

34. C. S. Osborne, L. Chakalova, K. E. Brown, D. Carter, A. Horton, E. Debrand, B. Goyenechea, J. A. Mitchell, S. Lopes, W. Reik, P. Fraser, Active genes dynamically colocalize to shared sites of ongoing transcription. Nat. Genet. 36, 1065-1071 (2004).

35. S. Schoenfelder, I. Clay, P. Fraser, The transcriptional interactome: Gene expression in 3D. Curr. Opin. Genet. Dev. (2010), , doi:10.1016/j.gde.2010.02.002.

36. S. Zhang, N. Übelmesser, N. Josipovic, G. Forte, J. A. Slotman, M. Chiang, H. J. Gothe, E. G. Gusmao, C. Becker, J. Altmüller, A. B. Houtsmuller, V. Roukos, K. S. Wendt, D. Marenduzzo, A. Papantonis, RNA polymerase II is required for spatial chromatin reorganization following exit from mitosis. Sci. Adv. (2021), doi:10.1126/sciadv.abg8205.

37. S. Schoenfelder, T. Sexton, L. Chakalova, N. F. Cope, A. Horton, S. Andrews, S. Kurukuti, J. A. Mitchell, D. Umlauf, D. S. Dimitrova, C. H. Eskiw, Y. Luo, C. L. Wei, Y. Ruan, J. J.

Bieker, P. Fraser, Preferential associations between co-regulated genes reveal a transcriptional 
interactome in erythroid cells. Nat. Genet. (2010), doi:10.1038/ng.496.

38. D. Sun, S. Roth, M. J. Black, A quantitative analysis of current practices in optical flow estimation and the principles behind them. Int. J. Comput. Vis. 106, 115-137 (2014).

39. B. Matérn, Spatial variation (Springer-Verlag, 1986). 


\section{Figure captions}

Figure 1: DFCC workflow. a) RNAP-Dendra2 stained nuclei of U2OS cells are imaged with a time interval of $\Delta \mathrm{t}$ by confocal microscopy. b) Flow fields between successive images are computed using Optical Flow. c) The spatial correlation in flow field direction (upper panel) and flow magnitude (lower panel) is computed over increasing space lags (averaged over the two spatial dimensions) and over accessible time lags (from blue to red). The spatial directional and magnitudinal correlation length, respectively, is obtained via a regression to the Whittle-Matérn covariance model for every time lag (insets).

Figure 2: Spatial correlation of RNAP and DNA dynamics in the absence of serum. a) Exemplary RNAP-Dendra2 stained nucleus. b) Directional and c) magnitudinal correlation length of RNAP and DNA over increasing time lag. Both DNA and RNAP show a weakly increasing directional correlation while RNAP's correlation in magnitude exceeds the magnitudinal correlation length of DNA in the absence of serum.

Figure 3: Spatial correlation of RNA Pol II upon serum-stimulation and DRB treatment. a) Directional correlation length of RNA Pol II in the absence and presence of serum stimulation. b) Analogous for the magnitudinal correlation length. c-d) Analogous for the magnitudinal correlation length.

Figure 4: Spatial correlation of RNA Pol II and DNA dynamics upon DRB treatment of the cell. a) Directional correlation length for DNA in the absence and presence of serum. b) Magnitudinal correlation for DNA in the absence and presence of serum. c-d) Superimposed directional (c) and magnitudinal correlation (d) correlation length for DNA (dashed lines) and RNA Pol II (solid lines). While DNA dynamics become spatially correlated upon serum stimulation, RNA Pol II's directional and magnitudinal correlation decreases slightly. e-h) Analogous for DRB treatment in the presence of serum. Upon stalling RNA Pol II at the initiation step by addition of DRB to the medium, RNA Pol II's directional correlation slightly increases, while the opposite trend is observed for DNA dynamics. In contrast, DRB treatment reduces the magnitudinal correlation length of both RNA Pol II and DNA. 

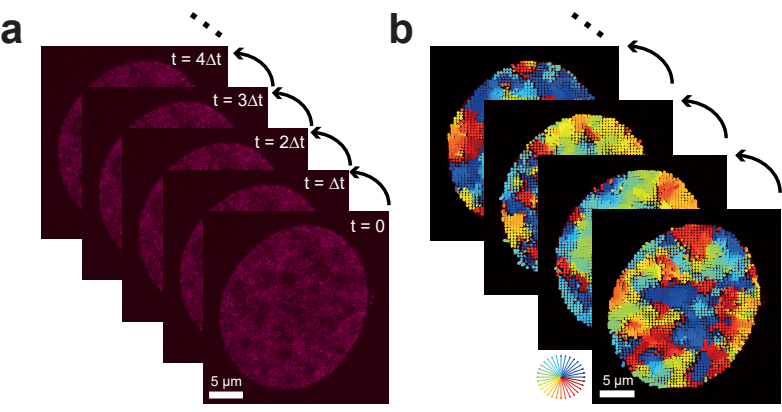

C

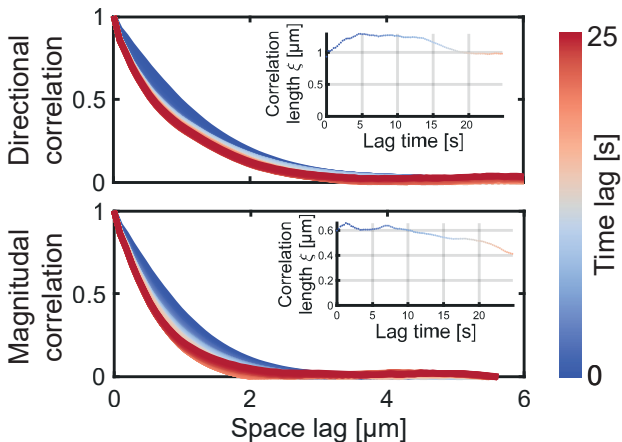


b Direction

$5 \mu \mathrm{m}$

을 ${ }^{\frac{\xi^{2}}{2}} 6$

U 这

0

5

10

15

Lag time [s]

C Magnitude

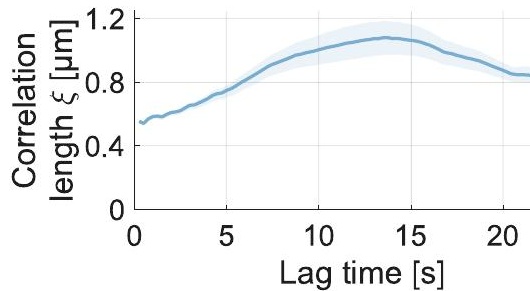


